\title{
Mitochondrial cytochrome redox states and respiration in acute pulmonary
}

\section{oxygen sensing}

\author{
N. Sommer*, O. Pak*, S. Schörner*, T. Derfuss", A. Krug" ${ }^{\#}$ E. Gnaiger, \\ H.A. Ghofrani*, R.T. Schermuly*, C. Huckstorf ${ }^{+}$, W. Seeger*, \\ F. Grimminger* and N. Weissmann*
}

ABSTRACT: Hypoxic pulmonary vasoconstriction (HPV) is an essential mechanism to optimise lung gas exchange. We aimed to decipher the proposed oxygen sensing mechanism of mitochondria in HPV.

Cytochrome redox state was assessed by remission spectrophotometry in intact lungs and isolated pulmonary artery smooth muscle cells (PASMC). Mitochondrial respiration was quantified by high-resolution respirometry. Alterations were compared with HPV and hypoxiainduced functional and molecular readouts on the cellular level. Aortic and renal arterial smooth muscle cells (ASMC and RASMC, respectively) served as controls.

The hypoxia-induced decrease of mitochondrial respiration paralleled HPV in isolated lungs. In PASMC, reduction of respiration and mitochondrial cytochrome $c$ and aa3 (complex IV), but not of cytochrome b (complex III) matched an increase in matrix superoxide levels as well as mitochondrial membrane hyperpolarisation with subsequent cytosolic calcium increase. In contrast to PASMC, RASMC displayed a lower decrease in respiration and no rise in superoxide, membrane potential or intracellular calcium. Pharmacological inhibition of mitochondria revealed analogous kinetics of cytochrome redox state and strength of HPV.

Our data suggest inhibition of complex IV as an essential step in mitochondrial oxygen sensing of HPV. Concomitantly, increased superoxide release from complex III and mitochondrial membrane hyperpolarisation may initiate the cytosolic calcium increase underlying HPV.

KEYWORDS: High-resolution respirometry, hypoxic pulmonary vasoconstriction, oxygen consumption, reactive oxygen species, spectrophotometry

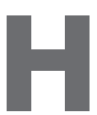
ypoxic pulmonary vasoconstriction (HPV) is an essential mechanism of the lung to match blood perfusion to alveolar ventilation, to optimise pulmonary gas exchange. Despite the early recognition of this mechanism, which prevents arterial hypoxaemia during regional alveolar hypoxia, the underlying $\mathrm{O}_{2}$ sensing and signal transduction processes have not been fully resolved. HPV occurs within seconds and has been found in vivo and in explanted lungs of different species to commence at mild hypoxia, in rabbit lungs at oxygen tension $\left(\mathrm{PO}_{2}\right)$ levels of $\leqslant 75 \mathrm{mmHg}$ $[1,2]$. For acute HPV, pulmonary artery smooth muscle cells (PASMC) have been suggested to represent the $\mathrm{O}_{2}$ sensor and effector cells. Upon exposure to hypoxia, isolated PASMC 1) constrict, 2) increase their intracellular calcium concentration and 3) decrease their plasma membrane potential difference. Such responses occur at $\mathrm{O}_{2}$ levels below a $\mathrm{PO}_{2}$ of $20-50 \mathrm{mmHg}$ [3-5]. Mitochondria, as the primary $\mathrm{O}_{2}$-consuming organelle of the cell, have long been thought to play a key role in the $\mathrm{O}_{2}$ sensing process of HPV, with recent new findings stimulating this field of research [6-9]. Mitochondria use oxygen for energy production by transferring electrons along an energy gradient onto oxygen. The transfer is achieved by different redox reactions of mitochondrial complex I, II, III and cytochrome c, until finally the electron is passed to oxygen at complex IV of the mitochondrial respiratory chain. However, simple inhibition of the last step of the respiratory system by hypoxia seemed not to be the mechanism of HPV, as 1) complex IV and mitochondria of isolated

\section{AFFILIATIONS}

*University of Giessen Lung Center (UGLC), Medical Clinic II, JustusLiebig-University,

"LEA Medizintechnik GmbH, Giessen,

+Institute of Physiology, University of Rostock, Rostock, Germany.

"Dept of Transplant Surgery, D. Swarovski Research Laboratory, Innsbruck Medical University, Innsbruck, Austria.

CORRESPONDENCE

N. Weissmann

University of Giessen Lung Center

Gaffkystrasse 11

35392 Giessen

Germany

E-mail: Norbert.Weissmann@

uglc.de

Received:

Jan 252009

Accepted after revision:

April 192010

First published online:

June 012010 
non-pulmonary cells are largely functional at very low oxygen concentrations (i.e. have high oxygen affinity) [10], but HPV occurs at mild hypoxia and 2) application of inhibitors of mitochondrial respiratory complexes suggested the oxygen sensing mechanism to be upstream of complex IV [7, 11, 12]. As mitochondria are also known to produce reactive oxygen species (ROS) as by-products during electron transfer, ROS production at complex I, II or III had been suggested as the oxygen sensing mechanism. However, there is currently much discussion about whether mitochondrial ROS release is increased or decreased during hypoxia to trigger HPV [1]. Underlying mechanisms have been proposed: 1) reduced ROS production by lack of $\mathrm{O}_{2}$ as a substrate, or reduced activity of the electron transfer system, or 2) increased ROS production by inhibition of complex III downstream of a suggested electron leak, or unspecific interference of $\mathrm{O}_{2}$ with the mitochondrial membrane [13, 14]. As a possible downstream sequence of such events, inhibition of voltage-gated potassium channels, activation of transient receptor potential channels, and/or other intra-/extracellular calcium channels, have been discussed as events leading to the contraction of precapillary PASMC, and thus HPV [1].

However, up to now it is not clear 1) if the mitochondrial electron transport system of PASMC is inhibited in the $\mathrm{O}_{2}$ range where HPV occurs; 2) if inhibition of the respiratory system occurs during HPV in the intact organ (isolated lung); 3) if there are changes in the redox state of mitochondrial cytochromes, which can be attributed to altered ROS production and HPV; and 4) if pulmonary cytochromes behave differently or similarly to those from the systemic circulation. With regard to the fact that, after birth, lung cells face a much higher $\mathrm{PO}_{2}$ than those of the rest of the body, such differences are to be anticipated. However, until now these uncertainties have not been resolved due to methodological limitations. In whole organs mitochondrial $\mathrm{O}_{2}$ affinity cannot be determined and, in isolated cells, simple determination of $\mathrm{O}_{2}$ affinity by $\mathrm{O}_{2}$ consumption measurements is hampered by artefacts due to non-mitochondrial oxygen consumption resulting in biphasic $\mathrm{O}_{2}$ kinetics during mild hypoxia [15]. To overcome these limitations, we first adapted the technique of remission spectroscopy to examine the redox state of the mitochondrial respiratory system in intact lungs and in isolated PASMC. This optical method allows determination of the redox state of specific cytochromes of mitochondria (cytochrome c, cytochrome $b_{1 / h}$ of complex III, cytochrome aa3 of complex IV) and, therefore, can provide information about hypoxic inhibition of mitochondrial respiration in isolated organs and cells. If electron transfer to oxygen is limited by hypoxia, cytochrome aa3 will become reduced, which is detected by a change in absorption characteristics (= difference spectrum). Moreover, measurement of the redox state of cytochrome $b_{1 / h}$ can provide insight into mechanism of ROS production. An increased electron transfer to produce superoxide could be reflected by an increased oxidation state. Secondly, we used high-resolution respirometry in PASMC, a technology which allows determination of hypoxic inhibition of mitochondrial respiration by calculation of $\mathrm{PO}_{2}$ at half-maximum respiration $\left(P_{50}\right)$ according to hyperbolic Michaelis-Menten fitting, independently of non-mitochondrial oxygen consumption [10]. Moreover, we deciphered possible differences between PASMC, aortic smooth muscle cells (ASMC) and renal arterial smooth muscle cells (RASMC), and used inhibitors of mitochondrial respiratory complexes to verify the spectrophotometric results in intact lungs. Finally, we correlated the hypoxia induced alteration of redox state and respiration with mitochondrial ROS generation, mitochondrial membrane potential, as well as cytosolic calcium concentration. With these highly sensitive methods we could show that, in contrast to previous assumptions, inhibition of mitochondrial respiration at complex IV is important for the oxygen sensing process in HPV. Moreover, our data suggests that increased ROS production at complex III and increased mitochondrial membrane potential are subsequent events initiating HPV.

\section{MATERIAL AND METHODS \\ Animals}

Chinchilla bastard rabbits of either sex $(2.5-3.2 \mathrm{~kg}$ body weight) were used for the investigations. All animal experiments were approved by the local Governmental Commission.

\section{Isolated pulmonary, renal and aortal arterial smooth muscle cells}

Smooth muscle cells were isolated from pulmonary precapillary arteries and cultured as described previously [16]. The protocol was adapted for isolation of smooth muscle cells of renal precapillary arteries. ASMC were dissected from isolated aorta after removal of the endothelial and adventitial cell layer. Cells from first and second passage were cultured and measured in Medium 199 with 10\% fetal calf serum.

\section{Isolated perfused and ventilated rabbit lung}

The model of isolated perfused rabbit lungs has been described previously [2].

\section{Remission spectrophotometry}

Measurement of remission difference spectra was performed by a spectrophotometric system using a CytoSPEC II (LEA Medizintechnik, Giessen, Germany). For collection of spectra we used a newly-developed fibre-optic light guide (LEA flat probe, LEA Medizintechnik) fixed at the surface of the lung. Measurements in cell suspension were performed in a closed chamber (Oxygraph-2k, OROBOROS INSTRUMENTS, Innsbruck, Austria) with integrated fibre-optic light guides from the CytoSPEC II.

Redox state of single cytochromes aa3, $b_{1 / h}$ and $c$ was determined by multi-wavelength least-squares fitting of reference spectra of the respective cytochrome in the sum difference spectrum (TechPlot Sftek, Braunschweig, Germany; fig. 1a). The reference absorption spectra for single cytochromes were determined in the closed chamber system. For technical details please refer to the online supplementary material.

\section{High-resolution respirometry and $\mathrm{O}_{2}$ kinetics}

Mitochondrial respiration was determined at $37^{\circ} \mathrm{C}$ by highresolution respirometry using an Oxygraph-2k (OROBOROS INSTRUMENTS). This system allows analysis of the $\mathrm{O}_{2}$ kinetics of cellular respiration (for specifications see online supplementary material). The $\mathrm{O}_{2}$ consumption compared to $\mathrm{PO}_{2}$ exhibited biphasic kinetics (fig. 1b). The linear part at high $\mathrm{O}_{2}$ concentrations can partially be attributed to decreased 

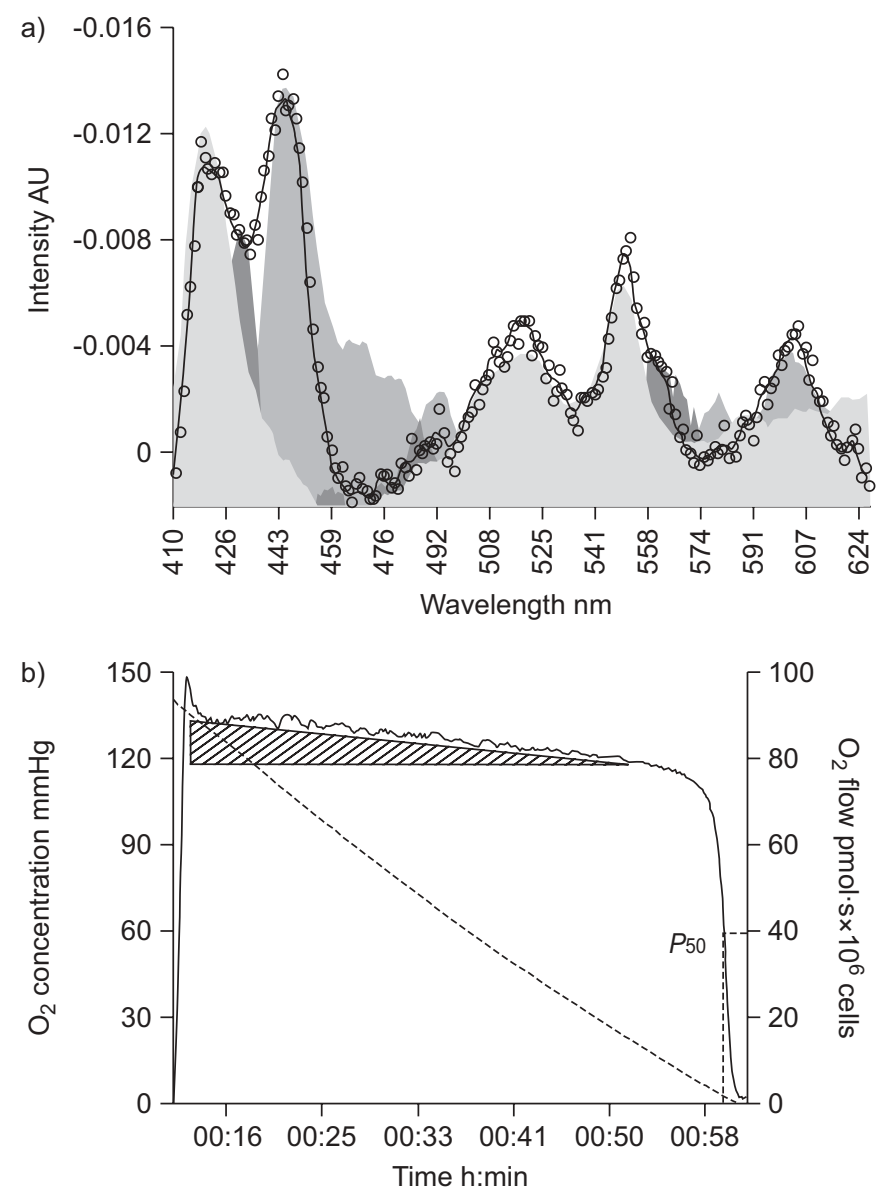

FIGURE 1. Deconvolution of remission spectra and determination of oxygen affinity. a) Fitting of spectra of single cytochromes in the measured difference spectrum (reduced minus oxidised spectrum) was performed for deconvolution. Negative intensity represents absorption ( $y$-axis) at specific wavelengths (x-axis). The wavelength-to-absorption curve (i.e. spectrum) depicts the sum spectrum of single cytochromes showing maximum absorption at specific wavelengths (cytochrome c ( $\square$; 420/550 nm), b//h $(\square ; 430 / 563 \mathrm{~nm})$, and aa3 ( $\square ; 445 / 603 /$ $605 \mathrm{~nm}$ ). Using least square-fitting, the spectrum of specific cytochromes was fitted into the measured sum spectrum and the level of absorption of each respective cytochrome was calculated in relation to the maximal signal (fully reduced spectrum). $\bigcirc$ : measured reduced spectrum; __ : fitted spectrum. For details see the Material and Methods section. b) The oxygen consumption in pulmonary arterial smooth muscle cells (-) was calculated by the first derivative of $\mathrm{O}_{2}$ concentration (-- ). The decrease in oxygen consumption due to non-mitochondrial $\mathrm{O}_{2}$ consumption and cell damage is depicted by the hatched area. Number of cells was $1.7 \times 10^{6} \mathrm{cells} \cdot \mathrm{mL}^{-1}$. For the calculation of oxygen pressure at half-maximum respiration (P50) refer to the Material and Methods section.

mitochondrial respiration due to loss of function and nonmitochondrial respiration, which illustrates that $\mathrm{O}_{2}$ consumption measurement alone does not allow for a complete description of the reaction of the mitochondrial respiratory system to hypoxia [15]. Therefore, the $\mathrm{O}_{2}$ affinity of the cells was studied in transitions from aerobic and anoxic states. The P50 was calculated according to the previously used method [10]. All measurements in cell suspensions were performed in intact cells in Medium 199.

\section{Mitochondrial membrane potential, ROS production and intracellular calcium}

Mitochondrial membrane potential was measured in a flow-through system in isolated PASMC using 5,5',6,6'tetrachloro-1,1',3,3'-tetraethylbenzimidazolylcarbocyanine iodide (JC-1) as a ratiometric indicator dye. Mitochondrial ROS production was measured with MitoSox and intracellular calcium with Fura-2. A detailed description is provided in the online supplementary material.

\section{Reagents}

The perfusate was purchased from Serag-Wiessner (Naila, Bayern, Germany). Medium 199, cyanide, antimycin A, sodium dithionite, cytochrome $\mathrm{c}$ and the $\alpha$-actin antibody were purchased from Sigma-Aldrich (St. Louis, MO, USA). VonWillebrand antibody was from Dako (Glostrup, Denmark), JC-1, MitoSox and Fura-2 from Invitrogen (Carlsbad, CA, USA).

\section{Statistics}

Results are presented as mean \pm SEM. A one-way ANOVA with Dunnet's post hoc test for the comparison of respiration at different $\mathrm{O}_{2}$ concentrations and evaluation of time course of ROS, mitochondrial membrane potential and intracellular calcium was employed, while a one-way ANOVA with the Newman-Keuls post hoc test was performed for the comparison of redox states of different cytochromes. An unpaired t-test was used for the comparison of two groups.

\section{RESULTS}

Isolated blood-free perfused lungs were ventilated at different $\mathrm{O}_{2}$ concentrations, ranging from an alveolar $\mathrm{PO}_{2}\left(\mathrm{~Pa}_{2} \mathrm{O}_{2}\right)$ of 0 $113 \mathrm{mmHg}$ for 10-min periods, alternating with 15-min periods of normoxic ventilation $\left(\mathrm{Pa}_{\mathrm{O}_{2}} 158 \mathrm{mmHg}\right)$. Increases in pulmonary arterial pressure $(\Delta P$ pa $)$ during hypoxic ventilation were detected at a $\mathrm{Pa}, \mathrm{O}_{2} \leqslant 75 \mathrm{mmHg}$ (fig. 2a). Recordings from parallel determinations of difference spectra by remission spectrophotometry revealed the first detectable changes at a $\mathrm{Pa}_{1} \mathrm{O}_{2}$ of $75 \mathrm{mmHg}$, reaching significance at $53 \mathrm{mmHg}$ at 410 $445 \mathrm{~nm}$ (fig. $2 \mathrm{~b}$ and c). Further alterations of remission spectra were evident at a $\mathrm{Pa}_{1} \mathrm{O}_{2}$ of $\leqslant 38 \mathrm{mmHg}$ in the range of 520 $590 \mathrm{~nm}$ and at a $\mathrm{Pa}_{1} \mathrm{O}_{2}$ of $0 \mathrm{mmHg}$ in the ranges of $410-460 \mathrm{~nm}$ and 500-630 nm (fig. $2 \mathrm{~b}$ and c). As reference, a difference spectrum after total reduction of mitochondrial cytochromes with cyanide, as well as reference spectra for cytochrome c $(420 / 550 \mathrm{~nm}), \mathrm{b}_{1 / \mathrm{h}}(430 / 563 \mathrm{~nm})$, and aa3 $(445 / 603 / 605 \mathrm{~nm})$ are provided. Recordings of remission spectra from PASMC under different $\mathrm{O}_{2}$ concentrations revealed similar alterations, such as in isolated lungs (fig. 3).

Fitting of reference spectra of single cytochromes into these spectra attributed the hypoxia-induced alterations to a reduction of mitochondrial cytochromes (fig. 4 and online supplementary material). While a reduction of cytochrome $b_{1 / h}$ was detectable only at $\mathrm{O}_{2}$ concentrations of $\leqslant 8 \mathrm{mmHg}$, cytochrome c was already reduced at a $\mathrm{PO}_{2}$ of $38 \mathrm{mmHg}$ in PASMC. For cytochrome aa3, reduction was observed at a $\mathrm{PO}_{2}$ of $\leqslant 23 \mathrm{mmHg}$. (fig. 4 a and b). The $\mathrm{O}_{2}$ dependence of redox alterations paralleled the inhibition in respiration determined by high-resolution respirometry (fig. 4a). Although cytochrome c was significantly reduced at higher oxygen levels $(38 \mathrm{mmHg})$ in PASMC than in ASMC or RASMC when compared with normoxia (fig. $4 \mathrm{~b}$ and fig. $4 \mathrm{a}$ in the online 

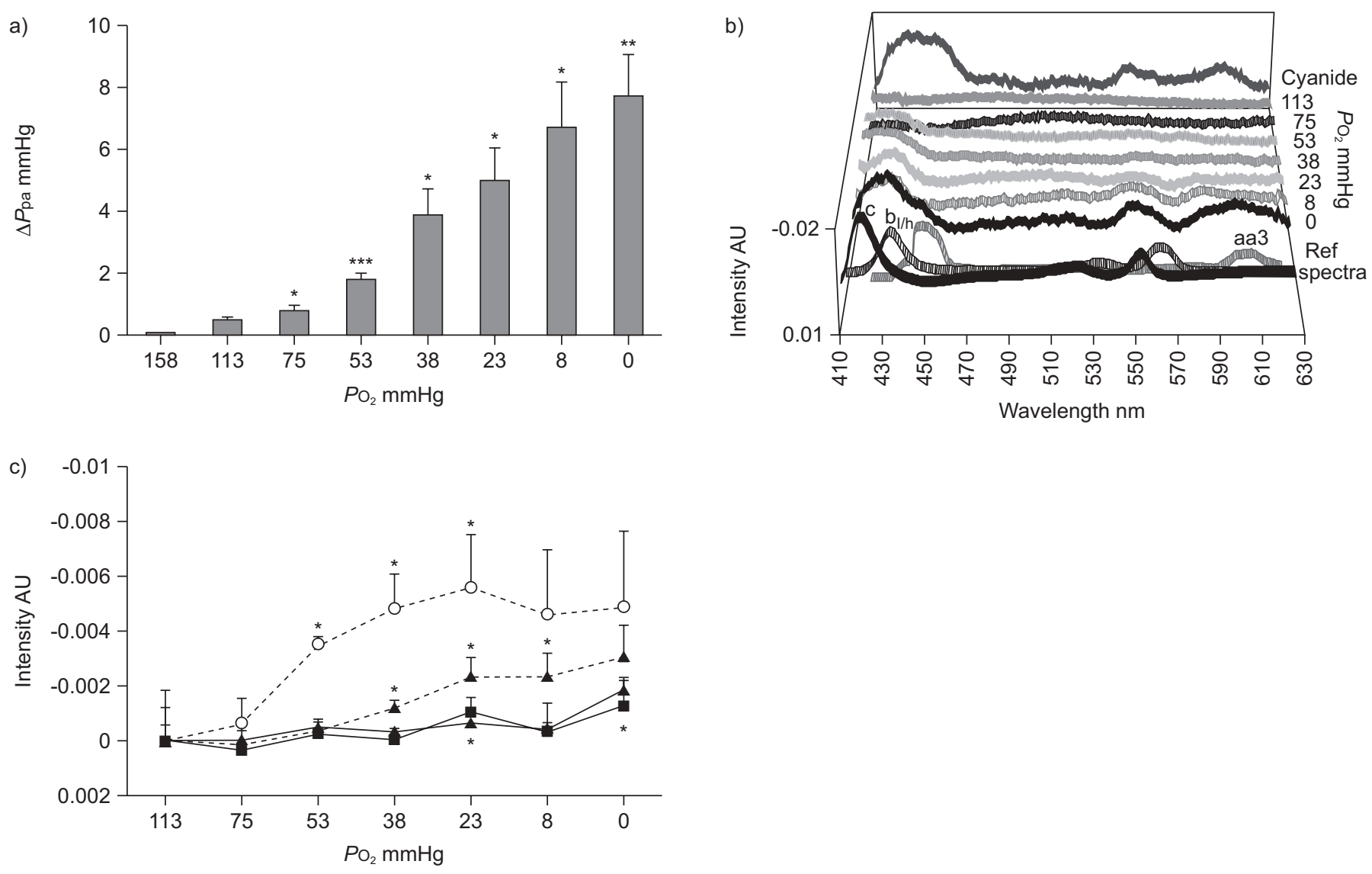

FIGURE 2. Oxygen dependency of the strength of hypoxic pulmonary vasoconstriction and corresponding alterations of remission spectra in the isolated rabbit lungs. a) Oxygen dependency of hypoxic pulmonary vasoconstriction (HPV). The strength of HPV is given as the maximum increase in the pulmonary arterial pressure ( $\triangle P$ pa) during 10-min periods of hypoxic ventilation for the depicted $\mathrm{O}_{2}$ concentration. Data are from five lung preparations. $*: p<0.05 ; * *: p<0.01 ; * \star *: p<0.001$ indicates significant differences compared with normoxic ventilation (oxygen tension $\left(\mathrm{PO}_{2}\right) 158 \mathrm{mmHg}$ ). b) Hypoxia-dependent alterations in remission spectra. Difference spectra were assessed by subtraction of normoxic from hypoxic spectra for repetitive hypoxic ventilation manoeuvres corresponding to (a). Spectra in the presence of cyanide (80 $\mu \mathrm{M})$ were recorded at the end of each experiment. Reference spectra for single cytochromes are depicted for comparison. Data are from five lungs each. c) Hypoxia-dependent

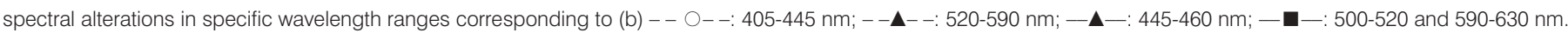
*: $p<0.05$ indicate significant differences compared to the calculated of $0 \%$ alterations at normoxia.

supplementary material), the absolute levels of hypoxiainduced reduction of mitochondrial cytochromes did not differ between PASMC, ASMC and RASMC (fig. 4b and fig. 4a in the online supplementary material). If any, only subtle changes between PASMC, ASMC and RASMC could be detected by a comparison of cytochrome $\mathrm{c}$ or of aa3 reduction in relation to that of cytochrome $b_{1 / h}$ within the different cell types. Such differences were significantly detectable in PASMC at 8 and $23 \mathrm{mmHg}$, in RASMC at severe hypoxia $(8 \mathrm{mmHg})$ and not in ASMC (\# in fig. 4b). A lower reduction of cytochrome $b_{1 / h}$ compared to cytochrome aa3 or c allows cytochrome $b_{1 / h}$ to still pass on the electrons at hypoxic levels, when electron transfer of cytochrome c or aa3 is already compromised due to low oxygen availability. Fully reduced spectra of PASMC, ASMC and RSMC differed in the wavelength range around $430 \mathrm{~nm}$ (fig. 4c). At anoxia difference spectra of PASMC minus RASMC revealed spectral alterations resembling reduced spectra of cytochrome b558 of nicotinamide adenine dinucleotide phosphate (NADPH) oxidase (fig. 4d). However at $7.5 \mathrm{mmHg}$ spectra of PASMC, ASMC and RASMC were not different. The first significant inhibition of respiration was found at $\mathrm{PO}_{2}$ levels of $\leqslant 105 \mathrm{mmHg}$ in PASMC (fig. 5a). The $P 50$ for PASMC was calculated to be a $P_{\mathrm{O}_{2}}$ of $0.84 \pm 0.03 \mathrm{mmHg}$, $0.82 \pm 0.05 \mathrm{mmHg}$ for ASMC, and $0.58 \pm 0.04 \mathrm{mmHg}$ for RASMC (fig. 5b). The onset of mitochondrial cytochrome reduction in PASMC correlated well with an increase in mitochondrial membrane potential, mitochondrial ROS production and the intracellular calcium concentration, with first detectable alterations occurring at a $\mathrm{PO}_{2}$ of $23 \mathrm{mmHg}$ (fig. 6a, b and c). Mitochondrial membrane potential was significantly increased in PASMC and ASMC, but not RASMC (fig. 6d). In contrast hypoxia induced an increase in mitochondrial ROS production and intracellular calcium in PASMC, but neither in ASMC nor in RASMC (fig. 6e and f). The increase of mitochondrial membrane potential and ROS production preceded the intracellular calcium increase in PASMC, the latter showing a similar time kinetics as found previously [16] (fig. $6 \mathrm{~g}, \mathrm{~h}$ and $\mathrm{i}$ ).

In the absence of cyanide, the strength of HPV was $3.0 \pm 0.5 \mathrm{mmHg}$, when challenging the lungs with hypoxia $\left(P a, O_{2} 23 \mathrm{mmHg}, \mathrm{n}=4\right)$. Inhibition of the mitochondrial 


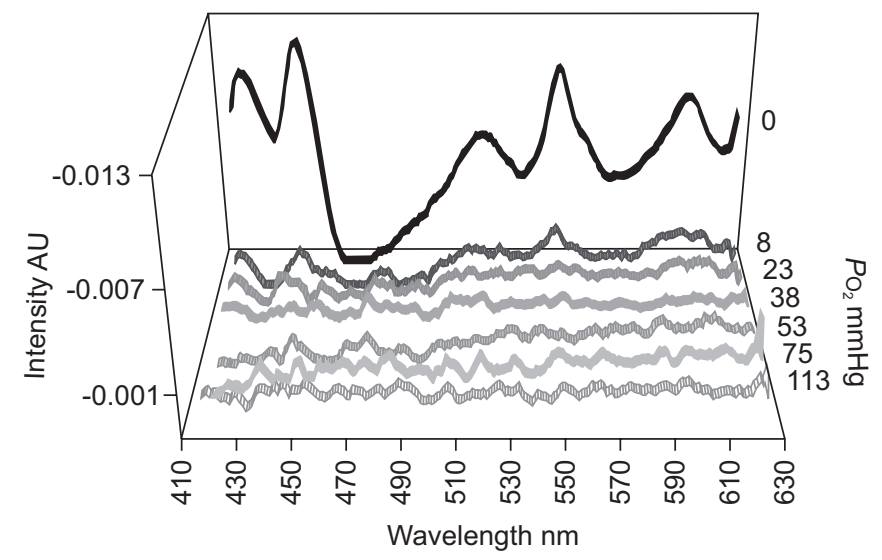

FIGURE 3. Oxygen-dependent alteration of remission spectra in isolated pulmonary arterial smooth muscle cells (PASMC). Difference spectra of hypoxic exposure of PASMC were assessed by subtraction of normoxic from hypoxic spectral values. Negative intensity (-) represent absorption peaks at specific wavelengths for single cytochromes (cytochrome c (420/550 nm), b b/h (430/ $563 \mathrm{~nm})$, and aa3 (445/603/605 nm; see fig. 1a). Fitting of single cytochromes to quantify absorption is performed in figure 4. Data are from nine experiments (six independent cell preparations).

respiratory system by cyanide in intact lungs inhibited HPV dose-dependently and reduced mitochondrial cytochromes with similar kinetics (fig. 7a). Cytochrome $b_{1 / h}$ was significantly less reduced than cytochrome aa 3 and $\mathrm{c}$ by cyanide treatment (fig. 7a). In parallel with inhibition of HPV cyanide induced a normoxic vasoconstriction determined as a $P$ pa increase of $0.8 \pm 0.2 \mathrm{mmHg}(\mathrm{n}=4)$. In isolated PASMC, cyanide inhibited maximal reduction of mitochondrial cytochromes and respiration less than HPV in isolated lungs (fig. $7 \mathrm{~b}$ and c). In isolated lungs, 2-n-heptyl-4-hydroxyquinoline-N-oxide (HQNO) reduced cytochrome $b_{1 / h}$ and oxidised cytochrome $c$ to a similar extent as it inhibited HPV (fig. 7d). Anoxic mitochondrial reduction was suppressed by HQNO in PASMC with an analogous dose-effect as for the inhibition of HPV in the isolated perfused lung (fig. 7e). However, such a correlation was not found for respiration (fig. 7f). Rotenone inhibited HPV, but resulted in no redox changes at a $\mathrm{PO}_{2}$ of $23 \mathrm{mmHg}$ in the isolated lung (fig. 7g). However, cytochrome reduction in PASMC correlated well with the inhibition of HPV in intact lungs (fig. 7h), whereas respiration was more inhibited than HPV (fig. 7i).

\section{DISCUSSION}

Although mitochondria have long been thought to be involved in the $\mathrm{O}_{2}$ sensing process underlying HPV $[7-9,12,13]$, the possible mechanisms of their contribution are not yet resolved. In particular, the high $\mathrm{O}_{2}$ affinity of mitochondria has been considered to exclude the inhibition of mitochondrial respiration as a possible mechanism underlying HPV [17]. Therefore, ROS production mainly at complex I and/or III by a yet unresolved mechanism has been proposed as an alternative pathway $[1,18,19]$. However, it is not clear whether mitochondria of the pulmonary circulation have a lower $\mathrm{O}_{2}$ affinity and whether differences in mitochondrial cytochromes redox state exist compared with mitochondria of the systemic microcirculation. Such differences may be anticipated, as the cells of the different vascular systems and thus their mitochondria have to be equipped to face different $\mathrm{PO}_{2}$ levels. Those of the systemic vasculature are exposed to a much lower $\mathrm{PO}_{2}$ than those of pulmonary resistance vessels after birth [20]. In addition, mitochondrial redox states can be modified by carbon monoxide [21] and nitric oxide [22], which may alter the $\mathrm{O}_{2}$ sensitivity in the intact organ. Against this background, we aimed to investigate the oxygen-dependent regulation of mitochondrial redox state and respiration in isolated lungs and PASMC. We adapted remission spectroscopy in intact, isolated and blood-free perfused rabbit lungs by construction of fibreoptic light guides to quantify redox alterations of mitochondrial cytochromes under graded hypoxia in the intact organ. As remission spectroscopy in intact lungs is limited by the fact that the sum spectra of all pulmonary cells are detected and PASMC are thought to be the sensor cells of HPV [23], we additionally measured respiration and cytochrome redox alterations in isolated PASMC by a combination of respirometry and spectrophotometry and compared this with hypoxia-induced cellular responses, such as mitochondrial matrix superoxide release, mitochondrial membrane potential, as well as intracellular calcium levels. Possible differences of ASMC or RASMC were determined.

Our findings suggest inhibition of complex IV as a prerequisite of mitochondrial oxygen sensing in HPV despite high oxygen affinity of mitochondria. We propose that such alterations initiate mitochondrial matrix superoxide release from complex III, an increase in mitochondrial membrane potential and finally an increase in cytosolic calcium. This concept is based on the facts that: 1) the hypoxia-induced decrease of mitochondrial respiration paralleled HPV in isolated lungs; 2) the reduction of cytochrome c and aa3 (complex IV) and respiration in PASMC correlated to the hypoxia-dependent increase in mitochondrial superoxide levels as well as mitochondrial membrane potential and intracellular calcium; 3) cytochrome $b_{1 / h}$ (complex III) was less reduced during hypoxia compared with cytochrome $\mathrm{c}$ and aa3; 4) these alterations were partially specific for PASMC, as RASMC displayed a lower decrease in respiration and no rise in matrix superoxide release, mitochondrial membrane potential and cytosolic calcium and there was a lower reduction of cytochrome $b_{1 / h}$ in relation to cytochrome aa3 and/or $\mathrm{c}$ in PASMC compared to ASMC or RASMC; and 5) pharmacological inhibition of mitochondria revealed analogous kinetics of cytochrome redox state and the strength of HPV.

Although in isolated lungs, mitochondrial inhibition determined by cytochrome aa3 (complex IV) reduction was only detected at a $\mathrm{Pa}_{\mathrm{a}_{2}} \mathrm{O}_{2}$ of $0 \mathrm{mmHg}$, mitochondrial respiration was inhibited already at a $\mathrm{PO}_{2}$ of $105 \mathrm{mmHg}$ in isolated PASMC, correlating to onset of HPV. This decrease in respiration was paralleled by reduction of cytochrome aa 3 and c in isolated PASMC. The alterations of mitochondrial cytochrome redox state reached significant levels at a $\mathrm{PO}_{2}$ of $38 \mathrm{mmHg}$ for cytochrome $\mathrm{c}$ and $23 \mathrm{mmHg}$ for cytochrome aa3, at which significant hypoxia-induced increase of mitochondrial superoxide, mitochondrial membrane potential and intracellular calcium release could also be detected. In this regard it has to be taken into account that measurements in the intact lung may be less sensitive due to the fact that redox signals not only from the sensor cells of HPV (PASMC), but also from non-sensor 

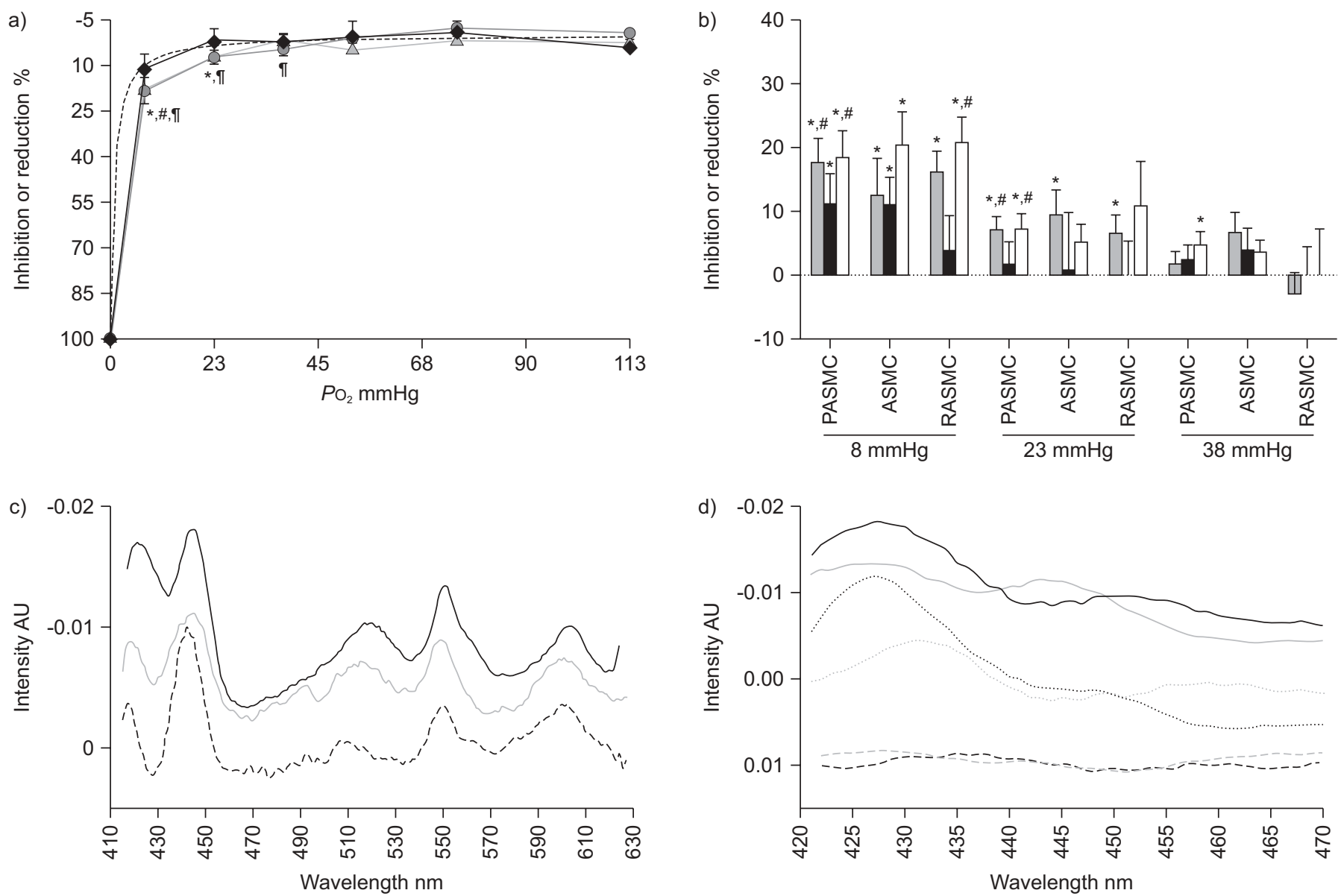

FIGURE 4. Oxygen dependency of redox alterations of cytochromes aa3, b, and c in pulmonary arterial, aortic and renal arterial smooth muscle cells (PASMC, ASMC and RASMC, respectively). Redox alterations were assessed from difference spectra by deconvolution. a) Reduction of cytochromes aa3 $(\mathbf{\Lambda})$, bl/h $(\bullet)$, c $(\bullet)$ in PASMC between 113-0 $\mathrm{mmHg}$. For comparison, the reduction in respiration from figure $5 \mathrm{a}$ is depicted (- - - ). Data are from $\mathrm{n}=16$ experiments from eight independent cell preparations. *. \#, $: \mathrm{p}<0.05$ indicate significant differences of cytochrome aa3, $\mathrm{b}_{\mathrm{l} / \mathrm{h}}$, and $\mathrm{c}$, respectively, compared with normoxia. b) Comparison of redox alterations to cytochrome aa3 $(\square), b_{1 / h}(\square)$ and $c(\square)$ in PASMC, ASMC and RASMC. Data are from at least $n=7$ individual experiments from at least $n=4$ independent preparations. *: $p<0.05$ indicate significant differences of cytochrome aa3, $b_{/ / h}$, or $c$ compared with normoxia. ${ }^{\#}: p<0.05$ indicate significant differences of cytochrome aa3 and $c$, compared with cytochrome $b_{1 / h}$. c) Fully reduced spectra at $0 \mathrm{mmHg}$ given for PASMC (-), ASMC (grey line) and RASMC (-- ). d) Difference spectra of PASMC minus ASMC as well as of PASMC minus RASMC at 0 mmHg and $7.5 \mathrm{mmHg}$. _- PASMC minus RASMC 0 mmHg; solid grey line: PASMC minus ASMC 0 mmHg; -----: PASMC minus RASMC $7.5 \mathrm{mmHg}$; dashed grey line PASMC minus RASMC $7.5 \mathrm{mmHg}$. For comparison spectra of cytochrome b558 (…..) and cytochrome bl/h (dotted grey line), derived from the addition of sodiumdithionite or antimycin $\mathrm{A}$ to completely reduce mitochondria or reduce cytochrome $\mathrm{b}_{/ / h}$, respectively, are given. $P_{\mathrm{O}_{2}}$ : oxygen tension

cells contribute to the determined spectra. The fact that the spectral changes seen in the isolated lung at a $\mathrm{Pa}_{3} \mathrm{O}_{2}$ of $75 \mathrm{mmHg}$ could not be observed in PASMC favours the suggestion that the onset of cytochrome reduction in the wavelength range of $410-440 \mathrm{~nm}$ in isolated lungs under mild hypoxia $\left(\mathrm{PO}_{2}\right.$ $75 \mathrm{mmHg}$ ) is not attributed to cytochrome $b_{1 / \mathrm{h}}$ reduction in PASMC, but rather related to other cytochromes of the $b$ class (haemoglobin, cytochrome b558) in other cell types.

At a $\mathrm{PO}_{2}$ of $23 \mathrm{mmHg}$, when first hypoxia-induced alterations in PASMC were seen, cytochrome c was reduced by $7.1 \pm 2.1 \%$, cytochrome aa 3 by $7.1 \pm 2.4 \%$ and respiration by $3.3 \pm 0.0 \%$. This level of hypoxia is well in line with the onset of HPV in the intact organ, since comparable $\mathrm{O}_{2}$ values have been suggested to occur at the level of precapillary PASMC in isolated lungs [20]. Furthermore, the range of onset of hypoxiainduced alterations resembles previous studies focussing on the onset of hypoxic cellular membrane depolarisation and intracellular $\mathrm{Ca}^{2+}$ increase [3, 4]. Therefore, we suggest that reduction of respiration and alterations of cytochrome redox state are initial steps of mitochondrial oxygen sensing in PASMC. The degree of inhibition of respiration seems too small for metabolic inhibition but may result in signal initiation, for example, by AMP-dependent protein kinase activation [8], regulation of mitochondrial calcium release [24] or ROS metabolism via regulation of cytochrome redox state. Cytochrome $b_{1 / h}$ exhibited a high oxidation state even at severe hypoxia, whereas cytochrome $\mathrm{c}$ and aa3 had already been reduced. Although cytochrome $b_{1 / h}$ has the lowest absorption coefficient according to our measurements, discernable changes were detectable according the equation for critical difference in the oxygen range of a $\mathrm{PO}_{2} \leqslant 23 \mathrm{mmHg}$. The higher oxidation state of cytochrome $b_{1 / h}$ suggests electron transfer directly onto oxygen, bypassing cytochrome c, resulting in 

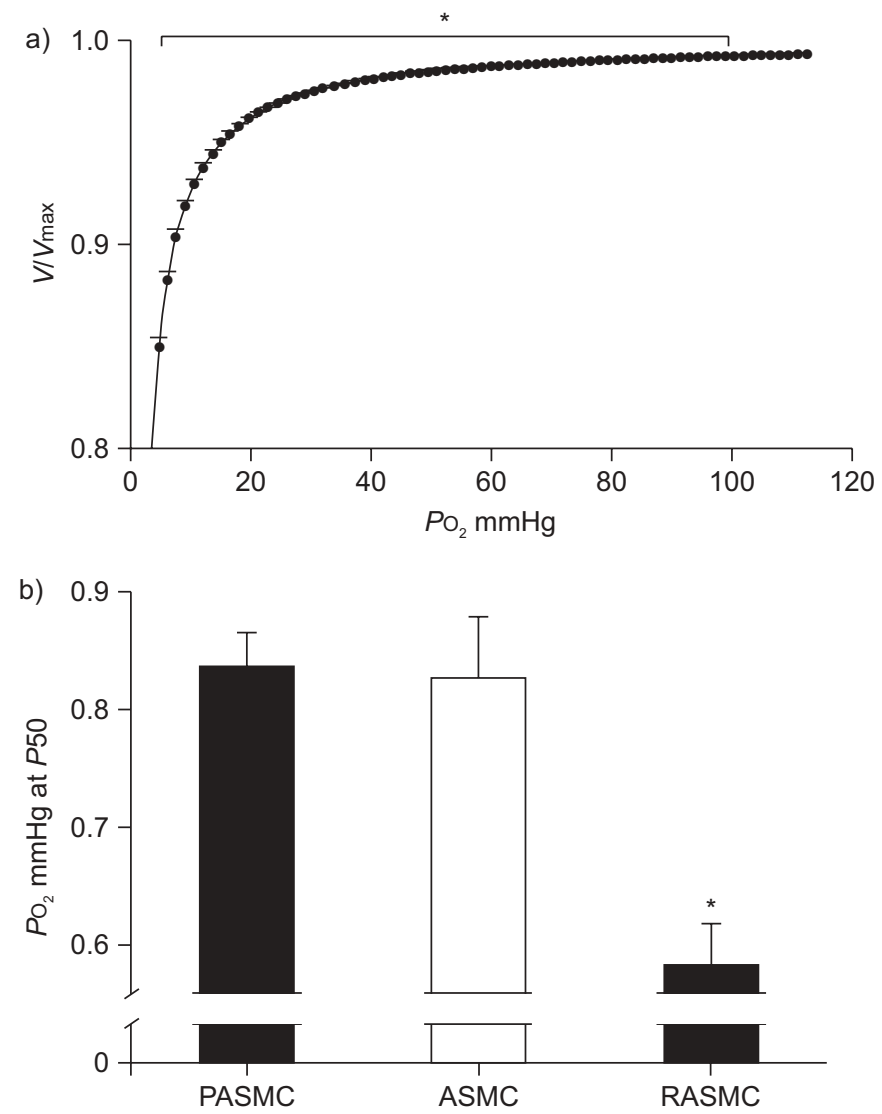

FIGURE 5. Oxygen dependency of respiration in pulmonary arterial smooth muscle cells (PASMC), aortic smooth muscle cells (ASMC) and renal arterial smooth cells (RASMC). a) Mean of original recording from PASMC. Respiration at different oxygen concentrations, calculated from oxygen pressure at half-maximal respiration $\left(P_{50}\right)$. The bar indicates significant decrease in respiration compared to an oxygen tension $\left(\mathrm{PO}_{2}\right)$ of $158 \mathrm{mmHg} .{ }^{*}$ : $\mathrm{p}<0.05$. b) Oxygen affinity of PASMC, ASMC and RASMC calculated as the oxygen concentration at half maximal respiration (P50; $\mathrm{n}=5-9) . V / V$ max: relative oxygen consumption in relation to maximal oxygen consumption. *: $p<0.05$.

superoxide production, as proposed by PALACIOS-CALLENDER et al. [25] in a monocyte cell line, demonstrating more pronounced reduction of cytochrome aa3 and c compared to cytochrome $b_{1 / h}$. This interpretation is supported by the detection of increased ROS production in PASMC found in our study. However, it has to be taken into account that the measurements of ROS with MitoSox are limited: 1) the function of this dye is dependent on mitochondrial membrane potential and 2) that mitochondrial matrix superoxide levels may not reflect those in the intermembrane space or the cytosol. Additional factors (e.g. membrane hyperpolarisation) or local regulation (e.g. local ROS scavenging) may also influence ROS release, as ASMC and RASMC also show lower reduction of cytochrome $b_{1 / h}$ at severe hypoxia, but no increase in ROS production. However, our finding is in accordance with hypoxia-induced increase in mitochondrial superoxide production at the semiubiquinone site at complex III and thus HPV as previously suggested [11, 14, 26-28]. Increased ROS levels may, in fact, regulate mitochondrial membrane potential via modulation of mitochondrial ion channels, although it has previously been shown in nonpulmonary cells that mitochondrial membrane hyperpolarisation may itself increase ROS [29]. Moreover, although it is not clarified yet how mitochondrial membrane hyperpolarisation is achieved, it is in line with previous studies [30].

The fact that both hypoxia-dependent alterations of respiration and the absolute level of cytochrome redox state in PASMC did not differ from those in ASMC (fig. 4b and fig. 4a in the online supplementary material) and the absolute level of cytochrome reduction did not differ between PASMC and RASMC (fig. 4b) allows for the conclusions that: 1) either inhibition of respiration is not the single mechanism underlying HPV; 2) that the difference in responses of PASMC and ASMC to hypoxia is caused by mechanisms downstream of a reduction in respiration, e.g. by different ion channel expression [31], or specific regulation of ion channel function [32]; or 3) with regard to ASMC, ASMC react similar to PASMC as they face rather high $\mathrm{PO}_{2}$ in vivo with the aorta carrying well oxygenated blood. The latter explanation is supported by the fact that, in contrast to ASMC, RASMC showed no hypoxia-induced membrane hyperpolarisation, which corresponds to higher respiration in hypoxia. A role of the culture conditions for the observed effects was largely excluded as only cells from passages 1 and 2 were used, no differences between these passages were detectable and cultured cells showed expected physiological responses, e.g. with regard to the hypoxia-induced intracellular calcium increase (see also fig. 2 in the online supplementary material).

Our data obtained with isolated cells confirm that hypoxiainduced spectral changes are detectable only in cytochromes of the mitochondrial electron transport system (see supplement) [33]. However, at anoxia differences in cytochrome spectra of PASMC and RASMC suggest a spectral contribution of cytochrome b558 of NADPH oxidase in PASMC. As no such difference was detected at higher oxygen concentrations, this supports the hypothesis that NADPH oxidases are active even at very low oxygen concentrations and thus their activity can also contribute to the regulation of HPV. This is in line with the previous finding in intact lungs [9].

Our data favour the conclusion that oxidised cytochrome $b_{1 / h}$ plays a role in HPV. Thus we inhibited cytochrome c oxidase by application of cyanide in intact lungs with parallel assessment of remission spectra. The decrease in the strength of HPV paralleled the degree of cytochrome reduction and thus mitochondrial inhibition. Again, cytochrome $b_{1 / h}$ was significantly less reduced than cytochrome c, suggesting that a differential regulation of redox states is possible and detectable also in the intact lung. In the presence of HQNO, an inhibitor of complex III, dose-dependent reduction of cytochrome $b_{1 / h}$ and oxidation of cytochrome $c$ correlated well with the inhibition of HPV in isolated lungs. Surprisingly, rotenone (inhibitor of complex I) did not result in any detectable changes of redox state at hypoxia of a $\mathrm{Pa}_{1} \mathrm{O}_{2}$ of $23 \mathrm{mmHg}$ in the isolated lung, although it inhibited HPV. However, as rotenone inhibits electron input in the respiratory chain, increased oxidation may only be detectable, when mitochondria are fully reduced [34]. Indeed, measurements in isolated PASMC showed inhibited reduction of mitochondrial cytochromes in anoxia which correlated with HPV. Also changes in the hypoxic redox state in presence of the other mitochondrial 

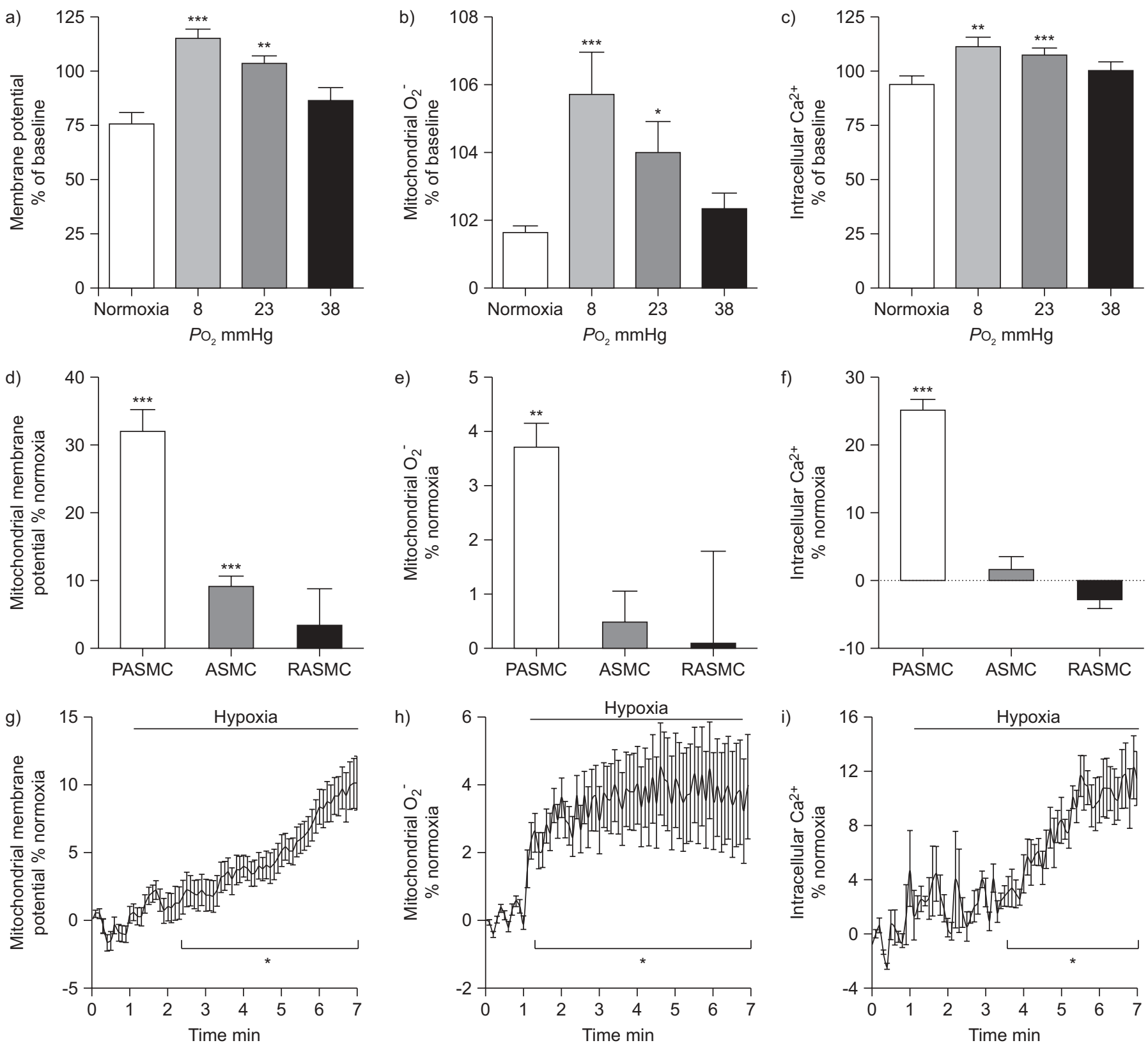

FIGURE 6. Hypoxia-, time- and cell type-dependent alterations of the mitochondrial membrane potential, mitochondrial superoxide, and intracellular calcium $\left(\mathrm{Ca}^{2+}\right)$ concentration. a) Hypoxia-dependent changes of pulmonary arterial smooth muscle cell (PASMC) mitochondrial membrane potential. b) Hypoxia-dependent alterations of mitochondrial superoxide concentration in PASMC. c) Hypoxia-dependent alterations of the cytosolic calcium concentration in PASMC. d) Comparison of the hypoxiadependent alteration of mitochondrial membrane potential in PASMC, aortic smooth muscle cells (ASMC) and renal smooth muscle cells (RASMC). Oxygen tension ( $P \mathrm{O}_{2}$ ) was reduced from normoxia to $8 \mathrm{mmHg}$. e) Comparison of the hypoxia-dependent alteration of mitochondrial superoxide concentration in PASMC, ASMC and RASMC. P $\mathrm{O}_{2}$ was reduced from normoxia to $8 \mathrm{mmHg}$. f) Comparison of the hypoxia-dependent increase in intracellular calcium in PASMC, ASMC and RASMC. $P \mathrm{O}_{2}$ was reduced from normoxia to $8 \mathrm{mmHg} . \mathrm{g})$ Time course of mitochondrial membrane potential in PASMC. Bar indicates hypoxia $(8 \mathrm{mmHg})$. h) Time course of mitochondrial superoxide production in PASMC. Bar indicates hypoxia ( $8 \mathrm{mmHg}$ ). i) Time course of intracellular calcium concentration in PASMC. Bar indicates hypoxia (8 $\mathrm{mmHg})$. Values for a)-f) are given as \% changes $6 \mathrm{~min}$ after induction of hypoxia, referenced to normoxic controls $\left(\mathrm{PO}_{2}=158 \mathrm{mmHg}\right)$. Values for $\left.\left.\mathrm{f}\right)-\mathrm{i}\right)$ are given as \% changes referenced to normoxic controls. $* * *: p<0.001$; ${ }^{* *}: p<0.01 ; *: p<0.05$ compared with normoxia. Data are from $n \geqslant 6$.

inhibitors correlated well with the alteration in the strength of HPV, again supporting the suggestion, that mitochondrial respiratory system activity is directly linked to HPV. Although our study could detect only subtle differences with regard to mitochondrial cytochrome reduction in PASMC when compared with ASMC and RASMC, a clear specificity for the pulmonary system was found with regard to respiration, mitochondrial superoxide release and membrane potential, and intracellular calcium release and thus, with regard to respiration and superoxide release, mitochondrial function. 

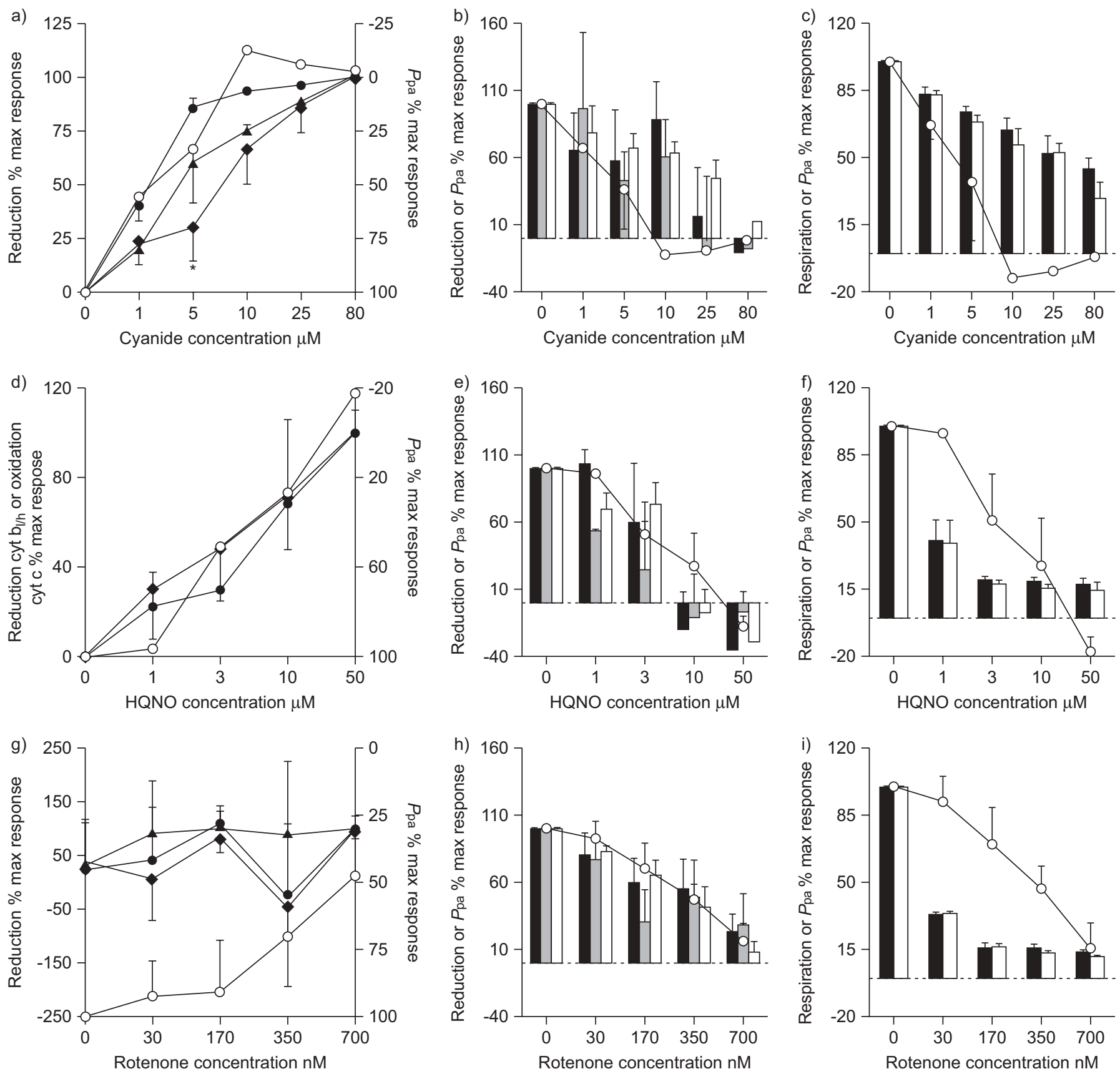

FIGURE 7. Effect of mitochondrial inhibitors on the strength of hypoxic pulmonary vasoconstriction (HPV), mitochondrial cytochrome reduction and mitochondrial respiration. a) Inhibition of HPV compared to the redox state of mitochondrial cytochromes at different cyanide concentrations in the isolated lung ( $\mathbf{\Lambda}:$ cytochrome aa3; cytochrome $\mathrm{b}_{/ / h} ;$ :cytochrome c; $\mathrm{O}: \mathrm{HPV}$ ). Data are given in \% of maximal reduction or maximum strength of HPV, respectively (\% max. response). *: significant difference compared to cytochrome $\mathrm{c}(\mathrm{p}<0.05)$. b) Inhibition of HPV compared to the maximal reduction of mitochondrial cytochromes at different cyanide concentrations in isolated pulmonary arterial smooth muscle cells (PASMC). - : cytochrome aa3; 1 : cytochrome $b_{/ / h} ; \square$ : cytochrome c; $\bigcirc: H P V$. c) Inhibition of HPV compared to mitochondrial respiration at different cyanide concentrations in isolated PASMC. $\mathbf{~ : ~ r e s p i r a t i o n ~ n o r m o x i a ; ~} \square$ : respiration $7.5 \mathrm{mmHg}$; $\mathrm{O}: \mathrm{HPV}$. d-f) As in parts a-c for different 2-n-heptyl-4hydroxyquinoline-N-oxide (HQNO) concentrations. $\mathrm{g}-\mathrm{i}) \mathrm{As}$ in parts a-c for different rotenone concentrations. $\mathrm{n}=3-4$ for redox measurements in isolated lungs and PASMC $\mathrm{n}=5$ for measurement of mitochondrial respiration. Ppa: pulmonary arterial pressure.

Even if the alterations in cytochrome reduction are not specific for the oxygen-sensing process in HPV, our data show that cytochrome reduction is a prerequisite for oxygen sensing and may explain mitochondrial ROS production mechanism. The interpretation of a specific role of cytochrome reduction suggested from our investigations on the cell level is supported by the findings from the inhibitor studies in the isolated lungs, correlating cytochrome reduction with HPV alterations.

In conclusion, we have established a new spectrophotometric technique and demonstrated, to the best of our knowledge for the first time, the $\mathrm{O}_{2}$ dependence of mitochondrial respiration 


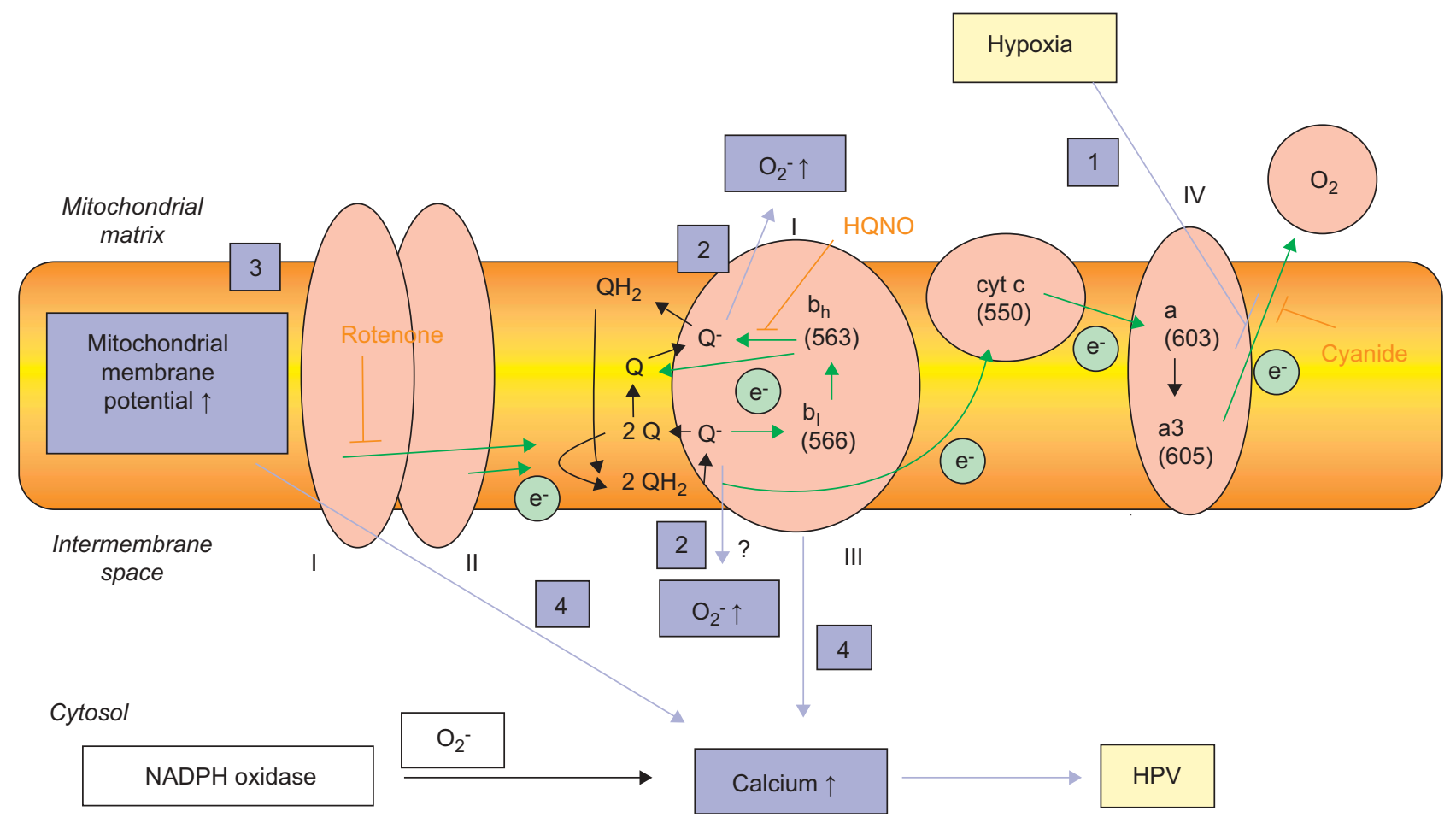

FIGURE 8. Role of mitochondrial complex IV in the oxygen sensing and signal transduction of hypoxic pulmonary vasoconstriction (HPV). Our data suggest that hypoxia inhibits electron transfer from complex IV to oxygen (1). As a possible result mitochondrial superoxide concentration (2) and mitochondrial membrane potential (3) increases. Finally the intracellular calcium concentration is elevated (4) and causes contraction of the pulmonary arterial smooth muscle cells (HPV). An increased superoxide production can originate from complex III caused by electron accumulation due to distal inhibition of electron flow at complex IV. In addition nicotinamide adenine dinucleotide phosphate (NADPH) oxidase-derived superoxide can contribute to the increase in intracellular calcium. $\mathrm{QH}_{2}$ : Ubiquinol; Q: Semiubiquinone; Q: Ubiquinone; HQNO: 2-nheptyl-4-hydroxyquinolone-N-oxide; cyt c: cytochrome c; I: complex I; II: complex II; III: complex III; IV: complex IV.

and redox state in isolated, intact lungs, PASMC, ASMC and RASMC.

As summarised in figure 8 , a small but significant decrease in mitochondrial respiration and reduction of cytochrome $\mathrm{c}$ and aa3 (complex IV) was found in the $\mathrm{O}_{2}$ range of HPV and/or hypoxic reactions of PASMC. Thus, despite the high oxygen affinity of mitochondria, inhibition of mitochondrial respiration may act as a sensor in acute pulmonary oxygen sensing by oxygen deprivation at the physiological electron transfer site (complex IV). This may result in an increased electron backup and leak at complex III, indicated by increased oxidation level of cytochrome $b_{1 / h}$, and subsequent ROS production increased mitochondrial membrane potential and cytosolic calcium levels. Alternatively, the latter events may be specifically regulated in PASMC independent from cytochrome redox state by mitochondrial respiration and subsequent downstream mechanisms. Moreover, ROS production from NADPH oxidases may interfere with mitochondrial signalling.

\section{SUPPORT STATEMENT}

This study was supported by EU 6th framework "Pulmotension" and Young Scientists support programme of the Justus-Liebig University, Giessen, Germany.

\section{STATEMENT OF INTEREST}

Statements of interest for T. Derfuss and A. Krug can be found at www. erj.ersjournals.com/site/misc/statements.xhtml

\section{ACKNOWLEDGEMENTS}

We thank R. Morty (University of Giessen Lung Center, Medical Clinic II, Giessen, Germany) for linguistic editing of the manuscript.

\section{REFERENCES}

1 Weissmann N, Sommer N, Schermuly RT, et al. Oxygen sensors in hypoxic pulmonary vasoconstriction. Cardiovasc Res 2006; 71: 620-629.

2 Weissmann N, Grimminger F, Walmrath D, et al. Hypoxic vasoconstriction in buffer-perfused rabbit lungs. Respir Physiol 1995; 100: 159-169.

3 Murray TR, Chen L, Marshall BE, et al. Hypoxic contraction of cultured pulmonary vascular smooth muscle cells. Am J Respir Cell Mol Biol 1990; 3: 457-465.

4 Olschewski A, Hong Z, Nelson DP, et al. Graded response of $\mathrm{K}^{+}$ current, membrane potential, and $\left[\mathrm{Ca}_{2}{ }^{+}\right]_{\mathrm{i}}$ to hypoxia in pulmonary arterial smooth muscle. Am J Physiol Lung Cell Mol Physiol 2002; 283: L1143-L1150.

5 Kang TM, Park MK, Uhm DY. Characterization of hypoxiainduced $\left[\mathrm{Ca}_{2}{ }^{+}\right]_{\mathrm{i}}$ rise in rabbit pulmonary arterial smooth muscle cells. Life Sci 2002; 70: 2321-2333.

6 Bonnet S, Michelakis ED, Porter CJ, et al. An abnormal mitochondrial-hypoxia inducible factor-1alpha-Kv channel pathway disrupts oxygen sensing and triggers pulmonary arterial hypertension in fawn hooded rats: similarities to human pulmonary arterial hypertension. Circulation 2006; 113: 2630-2641.

7 Waypa GB, Guzy R, Mungai PT, et al. Increases in mitochondrial reactive oxygen species trigger hypoxia-induced calcium responses in pulmonary artery smooth muscle cells. Circ Res 2006; 99: 970-978. 
8 Evans AM, Mustard KJ, Wyatt CN, et al. Does AMP-activated protein kinase couple inhibition of mitochondrial oxidative phosphorylation by hypoxia to calcium signaling in $\mathrm{O}_{2}$-sensing cells? J Biol Chem 2005; 280: 41504-41511.

9 Weissmann N, Zeller S, Schafer RU, et al. Impact of mitochondria and NADPH oxidases on acute and sustained hypoxic pulmonary vasoconstriction. Am J Respir Cell Mol Biol 2005; 34: 505-513.

10 Gnaiger E. Oxygen conformance of cellular respiration. A perspective of mitochondrial physiology. Adv Exp Med Biol 2003; 543: 39-55.

11 Weissmann N, Ebert N, Ahrens M, et al. Effects of mitochondrial inhibitors and uncouplers on hypoxic vasoconstriction in rabbit lungs. Am J Respir Cell Mol Biol 2003; 29: 721-732.

12 Leach RM, Hill HM, Snetkov VA, et al. Divergent roles of glycolysis and the mitochondrial electron transport chain in hypoxic pulmonary vasoconstriction of the rat: identity of the hypoxic sensor. J Physiol 2001; 536: 211-224.

13 Michelakis ED, Thebaud B, Weir EK, et al. Hypoxic pulmonary vasoconstriction: redox regulation of $\mathrm{O}_{2}$-sensitive $\mathrm{K}^{+}$channels by a mitochondrial $\mathrm{O} 2$-sensor in resistance artery smooth muscle cells. J Mol Cell Cardiol 2004; 37: 1119-1136.

14 Guzy RD, Schumacker PT. Oxygen sensing by mitochondria at complex III: the paradox of increased reactive oxygen species during hypoxia. Exp Physiol 2006; 91: 807-819.

15 Hutter E, Renner K, Jansen-Durr P, et al. Biphasic oxygen kinetics of cellular respiration and linear oxygen dependence of antimycin A inhibited oxygen consumption. Mol Biol Rep 2002; 29: 83-87.

16 Weissmann N, Dietrich A, Fuchs B, et al. Classical transient receptor potential channel 6 (TRPC6) is essential for hypoxic pulmonary vasoconstriction and alveolar gas exchange. Proc Natl Acad Sci USA 2006; 103: 19093-19098.

17 Bell EL, Emerling BM, Chandel NS. Mitochondrial regulation of oxygen sensing. Mitochondrion 2005; 5: 322-332.

18 Archer S, Michelakis E. The mechanism(s) of hypoxic pulmonary vasoconstriction: potassium channels, redox $\mathrm{O}_{2}$ sensors, and controversies. News Physiol Sci 2002; 17: 131-137.

19 Waypa GB, Schumacker PT. Hypoxic pulmonary vasoconstriction: redox events in oxygen sensing. J Appl Physiol 2005; 98: 404-414.

20 Marshall BE. Hypoxic pulmonary vasoconstriction. Acta Anaesthesiol Scand Suppl 1990; 94: 37-41.

21 D'Amico G, Lam F, Hagen T, et al. Inhibition of cellular respiration by endogenously produced carbon monoxide. J Cell Sci 2006; 119: 2291-2298.
22 Brown GC. Nitric oxide and mitochondria. Front Biosci 2007; 12 1024-1033.

23 Robertson TP. Point: release of an endothelium-derived vasoconstrictor and RhoA/Rho kinase-mediated calcium sensitization of smooth muscle cell contraction are/are not the main effectors for full and sustained hypoxic pulmonary vasoconstriction. J Appl Physiol 2007; 102: 2071-2072.

24 Ward JP, Snetkov VA, Aaronson PI. Calcium, mitochondria and oxygen sensing in the pulmonary circulation. Cell Calcium 2004; 36: 209-220.

25 Palacios-Callender M, Quintero M, Hollis VS, et al. Endogenous NO regulates superoxide production at low oxygen concentrations by modifying the redox state of cytochrome c oxidase. Proc Natl Acad Sci USA 2004; 101: 7630-7635.

26 Chen Q, Vazquez EJ, Moghaddas S, et al. Production of reactive oxygen species by mitochondria: central role of complex III. J Biol Chem 2003; 278: 36027-36031

27 Drose S, Brandt U. The mechanism of mitochondrial superoxide production by the cytochrome bc1 complex. J Biol Chem 2008; 283 21649-21654.

28 Erusalimsky JD, Moncada S. Nitric oxide and mitochondrial signaling: from physiology to pathophysiology. Arterioscler Thromb Vasc Biol 2007; 27: 2524-2531.

29 Murphy MP. How mitochondria produce reactive oxygen species. Biochem J 2009; 417: 1-13.

30 Michelakis ED, Hampl V, Nsair A, et al. Diversity in mitochondrial function explains differences in vascular oxygen sensing. Circ Res 2002; 90: 1307-1315.

31 Bonnet S, Archer SL. Potassium channel diversity in the pulmonary arteries and pulmonary veins: implications for regulation of the pulmonary vasculature in health and during pulmonary hypertension. Pharmacol Ther 2007; 115: 56-69.

32 Platoshyn O, Brevnova EE, Burg ED, et al. Acute hypoxia selectively inhibits KCNA5 channels in pulmonary artery smooth muscle cells. Am J Physiol Cell Physiol 2006; 290: C907-C916.

33 Hollis VS, Palacios-Callender M, Springett RJ, et al. Monitoring cytochrome redox changes in the mitochondria of intact cells using multi-wavelength visible light spectroscopy. Biochim Biophys Acta 2003; 1607: 191-202.

34 Lloyd D, Chance B. Electron transport in mitochondria isolated from the flagellate Polytomella caeca. Biochem J 1968; 107: 829-837. 